\title{
ARQUEOLOGÍA DEL CARIBE COSTARRICENSE. CONTRIBUCIONES CIENTÍFICAS. VOLUMEN 2. LUIS HURTADO DE MENDOZA (ED.). SAN JOSÉ: MASTER LITHO S. A., 2018
}

Jeffrey Peytrequín Gómez

\author{
Recibido: 28/04/2019 - Aceptado: 08/05/2019
}

Este texto se constituye en un verdadero esfuerzo continuado, ya que es el segundo peldaño de un proyecto -a largo plazo- que el editor en jefe de esta colección ha vislumbrado. De hecho, existen algunas comunicaciones entre los contenidos del volumen 1 y los del volumen 2, las cuales serán resaltadas (de forma general), de manera que permitan identificar ciertas tendencias en los aportes de esta serie para la arqueología nacional.

Al subrayar los hilos conductores convergentes, se reseñarán las distintas contribuciones de cada una de las personas autoras que participan en este volumen.

En el primer trabajo de Hurtado de Mendoza y Naranjo se establece una propuesta muy interesante para la clasificación y ubicación temporal de los petroglifos (el "modelo Guayabo"), esta -particularmente- aplicada a la cuenca del río Reventazón, donde se desarrolló un proyecto hidroeléctrico a cargo del Instituto Costarricense de Electricidad (ICE).

Llama la atención cómo, según los autores, el Período Formativo (2000-300 a. C.) es el que presenta la mayor proporción de petroglifos en la zona. Un dato fundamental, ya que, de forma contrastante, para las cuencas de los ríos San Carlos y Sarapiquí (en años recientes también muy exploradas -en el ámbito arqueológico- por parte del ICE) prácticamente no hay petroglifos que se podrían asociar con esas ocupaciones precolombinas tempranas. Lo anterior permite empezar a trazar una serie de comparaciones interregionales para una mejor comprensión del pasado precolombino de Costa Rica.

En general, la propuesta de Hurtado de Mendoza y Naranjo establece una nomenclatura específica de clasificación de petrograbados, un modelo taxonómico con intenciones de ser aplicado a escala regional y sugiere, a la vez, cierto procedimiento de datación de estas expresiones culturales pétreas.

Otro elemento por destacar de la propuesta clasificatoria es que contrasta con otras previas que han abordado este tópico, en el sentido que, de un modo explícito, 
sugiere que la distribución cultural de los petrograbados no es exclusiva de la última Fase de ocupación de la Subregión Arqueológica del Caribe Central, lo cual sería en este caso La Cabaña (800-1450 d. C.). Ello competiría con hipótesis precedentes que han señalado que la representación de grabados en piedra se da hasta que las sociedades antiguas se han "complejizado", es decir, solo ya muy avanzada la secuencia de las ocupaciones antiguas en el país.

Los autores son autocríticos al decir que existe una restricción clara del "modelo Guayabo" en cuanto a su aplicación a contextos extrarregionales e indican que este no funcionaría para otras regiones arqueológicas del país; ello por las particularidades culturales y el tipo de representación de los petrograbados que engloba. Si bien el modelo no, la técnica (para su construcción) podría ser adaptada para las otras regiones arqueológicas de Costa Rica y valorar así su potencial.

El segundo aporte corresponde a Ramírez, ella hace una muy buena y apretada síntesis de lo que fue su tesis de maestría académica (Ramírez). Aquí, además de estudiar las características particulares del sitio arqueológico Palmita, se compendia toda la información disponible acerca del Período Formativo no solo del país, sino a nivel regional. Precisamente con eso, se indican algunas tendencias interesantes sobre las relaciones sociales que hubo en ese momento de la Historia Precolombina.

Un aspecto fundamental, apoyado por los diferentes trabajos del proyecto hidroeléctrico Reventazón (en adelante PHR), es que la información reciente contradice la noción clásica (en la arqueología nacional) de considerar a las poblaciones del Formativo como dispersas y aisladas. Es decir, en la zona del Reventazón todos los datos son fehacientes de que ya había varias concentraciones poblacionales desde el Período Formativo y que, por lo tanto, eso no es un fenómeno exclusivo para Fases Culturales más "adelantadas" de la ocupación precolombina.

De destacar es la descripción que se hace sobre el tipo de estructuras habitacionales del sitio Palmita que se puede correlacionar, también, con los hallazgos y descubrimientos afines del sitio Sibon (Naranjo, 2014); lo cual ya establece ciertas tendencias de cómo -desde el Formativo- ya se empieza a incidir en el ambiente con algunas modificaciones y el aprovechamiento de recursos ${ }^{1}$ (algunas de esas prácticas trascienden y se pueden remontar incluso hasta el Período Paleoindio);" así como las características $\mathrm{y}$ formas en que se establecieron los asentamientos humanos en ese lapso. A la vez, en el volumen I de la serie hay un artículo de Naranjo (2016) sobre el último sitio mencionado e información de este calibre. De modo que aquí se da una conexión clara entre la primera entrega y la segunda de esta colección.

El tercer aporte es un trabajo sobre el sitio arqueológico Pantano (cuenca del Reventazón), un asentamiento con presencia de ocupaciones precerámicas y cerámicas de los Períodos Paleoindio y Arcaico (7000-2000 a. C.), aquí Hernández enfatiza respecto al componente Paleoindio de dicho sitio y sus características, lo cual permite también establecer un puente temático importante con el volumen I de la serie, 
particularmente con el artículo de Chávez acerca del sitio La Isla, de los sitios mejor conocidos del Paleoindio y que cuenta con la fecha radiocarbónica (absoluta) más temprana de toda la Historia Antigua de Centroamérica -12 400 a. C. (Chávez).

Volviendo al sitio Pantano, algo importante de subrayar es que como parte de su ocupación durante el Período Formativo no hay presencia de budares (amplios platos de cerámica con gruesos bordes), una característica típica de algunos de los sitios del Formativo en la región y en Costa Rica.

Más allá de rancias argumentaciones de corte difusionista (acerca de la distribución de los budares al norte y sur del país), es menester apuntar que dentro del espacio estudiado por el PHR (un área relativamente pequeña) existen sitios del Formativo cercanos con budares y otros que no cuentan con esta clase de artefactos. De tal modo, ya la explicación unicausal de que -simplemente- esto era un tema de distribución geográfica anclado en un asunto de difusión, con los datos generados por el PHR esa idea se desbanca.

La cuarta contribución es de Zúñiga, un reporte sobre un sitio multicomponente llamado Los Higuerones. ${ }^{3}$ Acá se sugiere que el modo de vida cazador se mantuvo fuertemente marcado todavía en tiempos cerámicos. Lo cual, si bien no es que nunca se haya indicado, no con datos tan concretos como los que se asocian a este sitio del Formativo (complejo cerámico Ujarrás): los asentamientos serían aun campamentos temporales compuestos por fogones y las huellas de postes, y por su ubicación, se pueden correlacionar con la posibilidad de que fueran estructuras livianas con una especie de contraviento.

Es síntesis, no todas las poblaciones locales durante el Formativo serían completamente sedentarias ni agrícolas, presentándosenos para ese período un panorama cultural mucho más variado (en cuanto al tipo de ocupaciones y las disposiciones económicas de subsistencia) de lo considerado hasta ahora.

El quinto aporte es un análisis dimensional sobre las ofrendas cerámicas de dos cementerios del sitio Juká, ${ }^{4}$ una muestra de 225 vasijas. Este trabajo conjunto, entre Hurtado Mendoza y Chávez, incursiona en el cálculo preciso de los volúmenes (litros) que tendrían los enseres cerámicos. Dicho esfuerzo permite que personas interesadas en tomar ese tipo de medidas, con las indicaciones que generan los autores (bastante didácticas de cómo estimar los índices), apliquen esto a más sitios arqueológicos y se pueda, entonces, realizar estudios comparativos que potencien una discusión al respecto.

Además, se sugiere que para el tipo cerámico Zoila Rojo Inciso hubo una especialización en cuanto a la manufactura (ligada a este) para formas asociadas a lo que serían escudillas. Para el caso de la Selva Arenoso Aplicado, esa especialización sería respecto a la elaboración de "vasos" y escudillas (de dimensiones medianas) y para la Selva Rojo sobre Agamuzado en relación a los "vasos" medianos.

Al igual que sucede con el volumen I de la serie, esta entrega cuenta con una sección trascendental: la de los "Informes Técnicos"; ya que en esta se exponen muchos 
datos brutos con una muy buena presentación gráfica y, de manera específica, aquí se socializa una gran cantidad de fechas de radiocarbono.

El primer informe técnico, a cargo de Gutiérrez, trata sobre un cementerio que se ubica en Guadalupe de Cartago, en la Lima, llamado Finca Piza, donde se recalibran (con el software más actualizado) unas fechas de radiocarbono que habían sido calibradas en el año 2012.

Finca Piza es un sitio de la Fase Cartago (800-1450 d. C.) donde había casi 30 tumbas de cajón concentradas. Como un dato importante, las 3 fechas de radiocarbono de este sitio permiten saber que, como mínimo, el cementerio fue utilizado durante 220 años seguidos. Es decir, es un cementerio relativamente pequeño (en cuanto a espacio utilizado y cantidad de tumbas), pero con un lapso temporal bastante extendido.

Por su parte en una de las tumbas, que por cierto es una de las que cuenta con fechamiento de $\mathrm{c}^{14}$, se presentó un acomodo mortuorio con un cuerpo extendido, el cual estaba ungido (los restos óseos) con pintura roja. Este tipo de hallazgos no son comunes en Costa Rica y, mucho menos, en el Valle Central. ${ }^{5}$

El último informe discute y contextualiza acerca de distintas fechas de carbono 14, distribuidas en quince muestras, las cuales fueron recuperadas en 6 sitios arqueológicos de la zona del Reventazón.

Destacan fechas que se ubican en lo que sería el Período Formativo para los sitios arqueológicos Palomo y Vista Lagunas, lo cual establece un rango bastante claro de ocupación entre el 770 y el 410 a. C. Los autores contrastan ello con previas fechas calibradas y controladas (dos sigma) del PHR; concluyendo que -precisamente- hay una marcada ocupación durante el Período Formativo en esa zona en un lapso de 400 años.

El resto de muestras de $\mathrm{c}^{14}$ lo que hacen es reforzar también algo muy interesante. Si bien existió un pico de ocupación durante el Formativo, del 800 al 400 a. C., en esa parte del país (por diversas razones que hay que estudiar más), esa ocupación -de alguna u otra forma- desciende un poco con el pasar de los años y se da otro pico de intensidad ocupacional en la Fase El Bosque, pero, principalmente marcado, en la transición de la Fase La Selva A a la Fase La Selva B (un rango del 200 a. C.-800 d. C.). En suma, lo que sugieren los datos es que hubo en esos tiempos una explosión demográfica en esa zona del país durante la ocupación precolombina.

Lo anterior, se contrasta con un descenso poblacional, o quizá un uso menos intensivo del espacio de la cuenca del Reventazón, ello en la última Fase de ocupación precolombina (La Cabaña, 1000-1450 d. C.).

Toda la información cronométrica disponible del PHR apunta hacia un panorama donde, prácticamente, para la Fase La Cabaña la cuenca del río Reventazón estaba siendo poco habitada. Al respecto, y como muestra un botón, está el caso concreto del 
sitio Manuel, de los pocos sitios que presentan manifestaciones claras o típicas de lo que sería el Período Tardío (en este hay ¡una única tumba de cajón!). ${ }^{6}$

Como se ha intentado subrayar, el texto Arqueología del Caribe costarricense, Contribuciones científicas, Volumen 2, aporta muchos nuevos datos de relevancia para la arqueología nacional; así como abre nuevas brechas para la discusión sobre las ocupaciones antiguas de este sector del país.

\section{Notas}

1 Esto de acuerdo con el estudio de fitolitos.

2 Del 13000 al 7000 a. C. aproximadamente.

3 Este cuenta con materiales asociados al Período Arcaico, Formativo y lo que sería la Fase La Selva A.

$4 \quad$ Fase La Selva (aprox. 300-800 d. C.).

$5 \quad$ Un antecedente directo de esto fue el hallazgo de Wallace y Accola $(1980,55)$ en el sitio Nacascolo (Guanacaste) de pigmento rojo de hematita esparcido sobre un enterramiento primario complejo; esto desde el tórax hasta los fémures, concentrado en la zona de la pelvis.

De los otros pocos sitios, con manifestaciones asociadas al último lapso de ocupación precolombina, en el área estudiada por el PHR están: San Antonio, Krikra y Pascua.

\section{Bibliografía}

Chávez, M. La Isla (L-251 LI): Un sitio del período Paleoindio en la cuenca media del Río Reventazón, Costa Rica. En Hurtado de Mendoza, L. (Ed.). Arqueología del Caribe Costarricense. Contribuciones Científicas Volumen 1 (pp. 13-24). Costa Rica, San José: Litografía e Imprenta LIL, S.A., 2016.

Naranjo, D. Áreas de actividad durante el período Formativo (2000-200 a.C.) en el sitio arqueológico Sibon (L-220 Sb), en Siquirres, Costa Rica (Tesis de licenciatura). Universidad de Costa Rica, San José, 2014.

Naranjo, D. Contextos arqueológicos durante el período Formativo (2000-200 a. C.) en el Caribe Central costarricense. Un acercamiento teórico y metodológico. En Hurtado de Mendoza, L. (Ed.). Arqueología del Caribe Costarricense. Contribuciones Científicas Volumen 1 (pp. 111-129). Costa Rica, San José: Litografía e Imprenta LIL, S. A., 2016.

Ramírez, M. Las dinámicas socioculturales de los pobladores del piedemonte de la cuenca baja del río Reventazón durante el Período Formativo (2000-300 a.C.): un enfoque desde el sitio arqueológico Palmita. Limón, Costa Rica (Tesis de maestría). Universidad de Costa Rica, San José, 2014.

Wallace, H. y R. Accola. Investigaciones arqueológicas preliminares de Nacascolo, Bahía Culebra, Costa Rica. Vinculos 6, 1-2 (1980): 51-65. 
Jeffrey Peytrequín Gómez. Costarricense. Universidad de Costa Rica, Escuela de Antropología, Profesor e Investigador Asociado.

Contacto: jeffrey.peytrequin@ucr.ac.cr

ORCID: 0000-0001-6837-3274 